\title{
Profilo farmacologico clinico e farmacoeconomico di un gel oftalmico a base di carbomero 974P REVIEW e PVA (Siccafluid®) nella terapia della cheratocongiuntivite secca
}

Lorenzo Pradelli*, Letizia Vacchini§

\begin{abstract}
BACKGROUND: The dry eye sindrome refers to a group of disorders of the tear film due to reduced tear production or excessive tear evaporation that is associated with symptoms of ocular discomfort and may cause disease of the ocular surface.

Dry eye syndrome varies in severity, duration and etiology.

The cornerstone of dry eye syndrome therapies includes the intraocular gel or ophthalmic solutions instillation, reaching correct artificial lubrication. Molecules that can produce a stable lubricating film are the carbomers.

METHODS: In the first step of our work we reviewed the data from literature reporting about carbomers' characteristics in respect to other lacrimal substitutes. Then, a pharmacoeconomical analysis has been performed on ophtalmic gels derived from carbomers 974P and PVA.

RESULTS: Dry eye sindrome, if not adequately treated, determines a deterioration of the patient's quality of life, other than high secondary costs.

CONCLUSION: The high therapeutical index of carbomers 974P and PVA-based gels, in addition to the their limited cost (totally free for Sjögren patients in Italy), suggests that this product is characterized by one of the best benefits-to-costs ratios in the treatment of dry eye sindrome.
\end{abstract}

Keywords: dry eye syndrome, carbomer, tear film

Farmeconomia e percorsi terapeutici 2005; 6 (3): 185-196

\section{INTRODUZIONE}

L'introduzione dei gel oftalmici a base di carbomeri, avvenuta nel 1989, ha rappresentato un importante progresso terapeutico nella gestione dei pazienti con sindrome da occhio secco, fino ad allora trattati esclusivamente con lacrime artificiali in soluzione e pomate oftalmologiche, dalle caratteristiche fisico-chimiche meno idonee.

In questo lavoro presentiamo un profilo farmacologico clinico e farmacoeconomico di un gel a base di carbomero 974P e alcol polivinilico (Siccafluid®) nel trattamento della sindrome da occhio secco.

\section{AMBITO DIUTILIZZO: LA SINDROME DA OCCHIOSECCO}

La cheratocongiuntivite secca, o sindrome da occhio secco, è una condizione patologica molto frequente, caratterizzata da un'inadeguata protezione della cornea da parte del film lacrimale. L'eziologia della sindrome è multifattoriale.

Dal punto di vista clinico l'alterazione delle lacrime può essere quantitativa (ipolacrimie), per insufficiente secrezione, tipica della sindrome di Sjögren e di altre connetivopatie di origine autoimmune, o qualitativa (dislacrimie), in cui l'alterata composizione del liquido lacrimale può dare origine a evaporazione eccessiva o a distruzione prematura del film. (Figura 1)

I due momenti patogenetici possono coesistere, per quanto ciascuno di essi sia in grado da solo di provocare la sintomatologia dell'occhio secco [1].

\section{Epidemiologia}

Le sindromi da occhio secco rappresentano una condizione relativamente frequente, particolarmente nella popolazione anziana, con prevalenza maggiore nel sesso femminile. La
* Centro di ricerche farmacoeconomiche Advanced Research Srl

§Gruppo Multimedica Milano 
Figura 1

Classificazione diagnostica dell'occhio secco (modificata da [1])

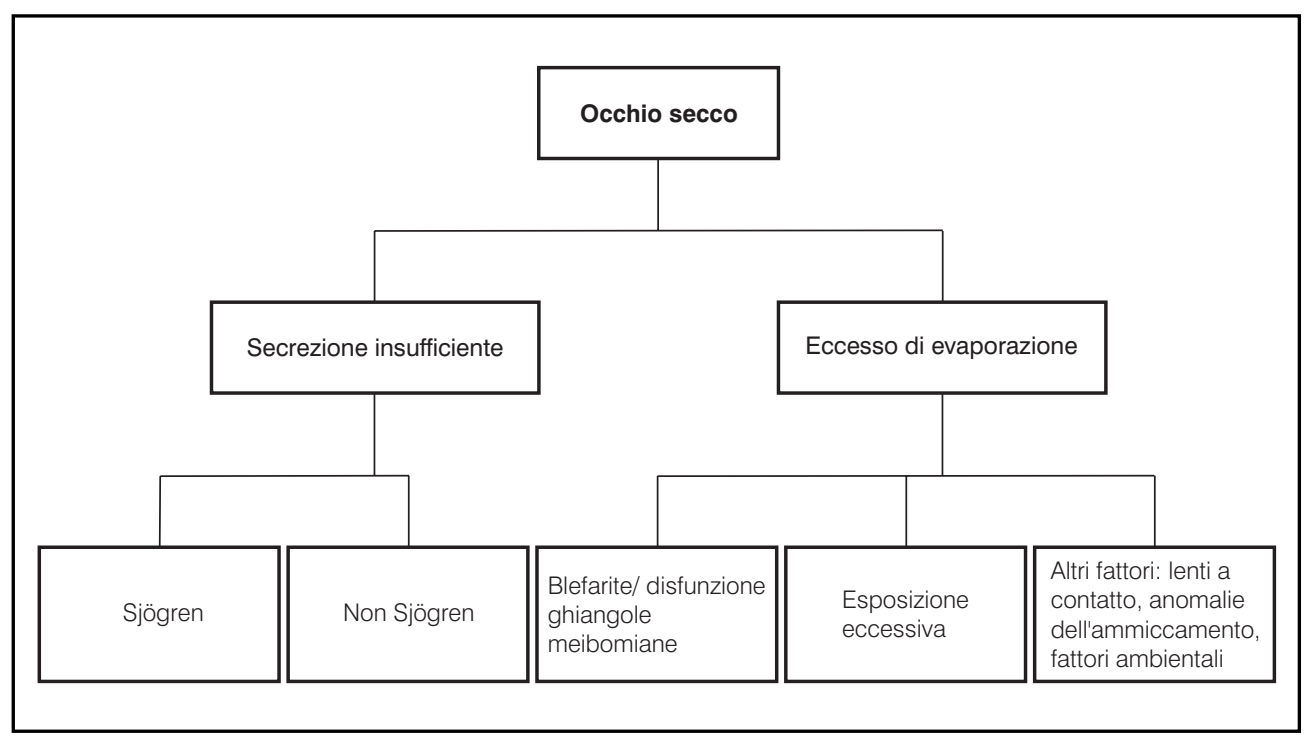

qualità dei dati epidemiologici sulla sindrome da occhio secco è limitata dalla difformità dei criteri diagnostici e dal fatto che nessun esame singolo o batteria di test è in grado di accertare o di escludere in maniera categorica la diagnosi di sindrome da occhio secco [1], ma non vi è alcun dubbio che si tratti di una condizione frequente con un impatto che varia da un lieve fastidio alla disabilità parziale. Le fonti sulla prevalenza della sindrome sono essenzialmente di due tipi: quelle basate su dati ottenuti nel corso di indagini cliniche e quelle derivate da analisi sulla popolazione generale. Il primo tipo di dati conferma la sua elevata frequenza nella popolazione mondiale dei pazienti oftalmici (17\% di 2.127 nuovi pazienti in uno studio giapponese) [2]; tali prevalenze potrebbero non riflettere la situazione nel complesso della popolazione. In uno studio epidemiologico statunitense, condotto su un campione di oltre 2.500 pazienti ultrasessantenni non selezionati in base a particolari caratteristiche cliniche, la prevalenza è risultata quasi del $15 \%$ in base a criteri clinici e del 3,5\% se per la formulazione della diagnosi di occhio secco venivano presi in considerazione, oltre alla sintomatologia, i risultati patologici ai test obiettivi (Schirmer $=5 \mathrm{~mm}$ o score $=5$ dopo colorazione al Rosa Bengala). Estrapolando questi valori al complesso della popolazione anziana degli USA, gli autori hanno stimato un numero di pazienti compreso tra 1 e 4,3 milioni, a seconda della percentuale utilizzata, solo per quanto riguarda gli ultrasessantenni [3].

Un altro studio di popolazione condotto a Melbourne (Australia), su un campione di 926 pazienti tra 40 e 97 anni ha utilizzato un valore soglia più alto per definire la diagnosi (Schirmer $\leq 8 \mathrm{~mm}$ o score $\geq 4$ dopo colorazione al Rosa Bengala), ottenendo valori di prevalenza maggiori: $16,3 \%$ o $10,8 \%$, a seconda del test utilizzato [4].
Infine, la percentuale di pazienti che riferiva di soffrire di occhio secco nel Beaver Dam Eye Study era del $8,4 \%$ tra i pazienti con meno di 60 anni e del 19\% negli ultraottantenni, con un valore medio complessivo del 14,4\% [5].

\section{Fisiopatologia e patogenesi}

Il film lacrimale è composto da tre strati, sovrapposti dall'esterno verso l'interno nell'ordine seguente:

a) uno strato lipidico, spesso $0,11 \mu \mathrm{m}$, prodotto dalle ghiandole di meibomio;

b) uno strato acqueo, spesso $7 \mu \mathrm{m}$, prodotto dalle ghiandole lacrimali principali e accessorie di Krause e Wolfring;

c) uno strato mucinico idrofilo, spesso 0,02$0,05 \mu \mathrm{m}$, prodotto dalle cellule caliciformi della congiuntiva.

Le anormalità che colpiscono anche uno solo dei tre strati (Tabella I) provocano l'instabilità del film lacrimale, e conseguentemente la sintomatologia dell'occhio secco. Lo strato lacrimale più frequentemente alterato è quello centrale, con prevalenza di iposecrezione lacrimale [6].

Le visioni più recenti tendono a considerare la superficie oculare e le ghiandole lacrimali come un'unità funzionale integrata, preposta al mantenimento di un film lacrimale integro mediante l'eliminazione delle lacrime "usate" e la loro sostituzione con liquido fresco. Una patologia o un malfunzionamento che colpisca questa unità funzionale causa l'instabilità e il mancato ricambio del film lacrimale, dando origine all'irritazione oculare e alla patologia infiammatoria epiteliale che costituiscono il quadro della cheratocongiuntivite secca.

Tra le cause di malfunzionamento di questo sistema ci sono l'invecchiamento, la diminuzione di fattori stimolanti come gli ormoni androgeni, malattie infiammatorie sistemiche 
come l'artrite reumatoide, patologie della superficie oculare come lo zoster oftalmico, malattie o farmaci con azione anticolinergica e altre.

\section{Clinica}

I sintomi più frequentemente lamentati dai pazienti con sindrome da occhio secco sono sensazione di sabbia o corpo estraneo, secchezza e bruciore che normalmente peggiorano verso sera, iperemia congiuntivale soprattutto nel quadrante inferiore, secrezione mucosa, irritazione oculare che peggiora negli ambienti fumosi, secchi o surriscaldati e durante gli sforzi prolungati (lettura, lavoro al computer) e l'ipersecrezione lacrimale riflessa. Questi sintomi sono presenti singolarmente o in combinazione, a seconda della tipologia e della gravità del quadro oculare.

Dal punto di vista obiettivo i segni rilevabili esternamente e mediante esame con lampada a fessura sono: riduzione del menisco lacrimale, aumento di detriti nel film, "pieghettatura" (pleating) congiuntivale, cheratopatia puntata superficiale, e difetti epiteliali (evidenziabili dopo colorazione con fluorescina), che nei casi più gravi possono dare origine a ulcerazioni corneali, iperemia e sofferenza congiuntivale (valutate grazie alle colorazioni con Rosa Bengala e Verde di Lissamina), presenza di placche o secrezione mucosa.

L'esame strumentale più utile è il test di Schirmer, che viene eseguito con una striscia di carta assorbente posta nella caruncola (valori inferiori a $5 \mathrm{~mm}$ in 5 minuti senza anestesia o inferiori a $10 \mathrm{~mm}$ con anestesia sono considerati anormali). In caso di valori anormali, è utile il test di Schirmer II, in cui la secrezione lacrimale viene valutata dopo l'applicazione di uno stimolo irritativo alla mucosa nasale (i valori inferiori a $15 \mathrm{~mm}$ in 5 minuti sono anormali).Un cenno particolare merita anche l'esame del tempo di rottura del film lacrimale (tear Break Up Time test o BUT), eseguito con l'instillazione di fluorescina e l'osservazione del film lacrimale alla lampada a fessura. Il tempo intercorso tra la stabilizzazione del film lacrimale dopo l'applicazione e la comparsa della prima discontinuità evidente è il tempo di rottura, considerato anormale se inferiore ai 10 secondi. Un BUT anormalmente basso è riscontrabile sia nelle ipolacrimie sia nelle dislacrimie da disfunzione delle ghiandole meibomiane.

Per quanto la diagnosi venga normalmente effettuata alla lampada a fessura, in qualche caso possono risultare utili alcuni esami di laboratorio, come l'analisi delle proteine lacrimali per la misurazione del contenuto in lisozima (normalmente $20-40 \%$ del contenuto proteico totale), l'analisi della lattoferrina o la citologia, che può evidenziare metaplasia squamosa e perdita delle cellule caliciformi [1].

\section{A carico dello strato acquoso}

Idiopatico

Alacrimia congenita

Ipovitaminosi A (xeroftalmia)

Ablazione delle ghiandole lacrimali

Denervazione sensoriale

Collagenopatie con interessamento vascolare, tra cui AR, granulomatosi di Wegener e LES

Sindrome di Sjögren primaria e secondaria (associata a AR, sclerodermia, polimiosite, poliarterite nodosa, tiroidite di Hashimoto, cirrosi epatobiliare cronica, polmonite interstiziale linfocitica, porpora trombocitopenica, ipergammaglobulinemia, macroglobulinemia di Waldenström, ecc.)

Cicatrizzazioni corneali da pemfigoide oculare, sindrome di Stevens-Johnson, ustioni chimiche e fisiche, atopia, tracoma

Farmaci, tra cui contraccettivi orali, antistaminici, betabloccanti, fenotiazine, atropina

Infiltrazione sarcoidotica o tumorale e fibrosi delle ghiandole lacrimali

\section{A carico dello strato lipidico}

Blefarite

Rosacea

A carico dello strato mucinico

Ipovitaminosi A

Tracoma

Cheratocongiuntivite difterica

Patologie muco-cutanee

Farmaci topici

Tabella I

Cause di alterazione del film lacrimale

\section{Patologie associate}

Le patologie sistemiche che si associano con maggiore frequenza alla sindrome da occhio secco sono la sindrome di Sjögren e la rosacea.

La sindrome di Sjögren è caratterizzata dalla contemporanea presenza di secchezza degli occhi (xeroftalmia) e della cavità orale (xerostomia); colpisce prevalentemente il sesso femminile, che rappresenta circa il $90-95 \%$ della casistica. La sindrome viene classificata in due sottotipi:

- pazienti con disfunzione immunitaria sistemica, ma senza evidenza di connetivopatia (sindrome di Sjögren primaria);

- pazienti con connetivopatia autoimmune, prevalentemente artrite reumatoide (sindrome di Sjögren secondaria).

Dal punto di vista istopatologico, tutti i casi sono caratterizzati dalla progressiva infiltrazione delle ghiandole lacrimali e salivari da parte di linfociti (principalmente B e CD4), con conseguente disorganizzazione della struttura tissutale e perdita di funzione. Per fare diagnosi di Sjögren, è necessario il riscontro di valori anormalmente bassi ( $\leq 5 \mathrm{~mm}$ in 5 minuti) al test di Schirmer, di evidenza obiettiva di flusso salivare ridotto (flusso salivare basale $\leq 1,5 \mathrm{ml}$ in 15 minuti), di evidenza bioptica di infiltrazio- 
ne linfocitica delle ghiandole salivari minori e di disfunzione immunitaria caratterizzata dalla presenza di autoanticorpi sierici tipici (anti-SSA (Ro) e anti SSB(La)).

La rosacea è una patologia della cute e degli occhi che colpisce prevalentemente i soggetti di carnagione chiara, per quanto non risparmi nessuna razza. I segni facciali caratteristici della rosacea comprendono eritema, teleangectasie, papule, pustole, ghiandole sebacee prominenti e rinofima. La disfunzione delle ghiandole meibomiane si sviluppa nella maggioranza di questi pazienti, che spesso presentano anche cheratocongiuntivite secca e blefarite marginale [1].

\section{Storia naturale}

La sindrome da occhio secco varia notevolmente per gravità, durata ed eziologia. Nella maggioranza dei pazienti, non si tratta di una condizione in grado di mettere in pericolo la funzionalità visiva, ma si caratterizza prevalentemente per la fastidiosa sintomatologia da irritazione. In taluni pazienti i fattori esacerbanti, quali l'assunzione di farmaci che riducono la secrezione lacrimale o l'esposizione a condizioni ambientali in grado di aumentare l'evaporazione, portano a un improvviso aumento della sintomatologia che li spinge a consultare l'oculista. Il venir meno dei fattori scatenanti spesso conduce a un netto miglioramento della sintomatologia e, in alcuni casi, può perfino essere curativo. In altri pazienti, in cui la sindrome è provocata da una iposecrezione lacrimale irreversibile o da una patologia cronica che aumenta l'evaporazione, come la blefarite, la patologia può acquisire carattere cronico, con sintomatologia remittente-recidivante o gradualmente ingravescente. Nei pazienti con forme da moderate a gravi, si possono sviluppare una metaplasia squamosa ed erosioni puntiformi dell'epitelio corneale, normalmente reversibili. Solo raramente i pazienti con forme molto severe di cheratocongiuntivite secca sviluppano complicazioni quali la cheratinizzazione della superficie oculare, ulcerazioni corneali sterili o infette, cicatrizzazione, assottigliamento o neovascolarizzazione corneale. In casi più rari, ma non eccezionali, la cheratolisi sterile e la cheratite infettiva possono esitare in perforazione della cornea con importante deficit visivo [1].

\section{Trattamento}

Il cardine della terapia dell'occhio secco è costituito dalla lubrificazione sostituiva, ottenuta con l'instillazione intraoculare di gel o soluzioni oftalmiche.

La terapia razionale della sindrome da occhio secco, tuttavia, deve tenere in considerazione il fatto che la secchezza oculare è una patologia che riguarda l'intera superficie ocu- lare, per cui va affrontata con un trattamento rivolto verso tutte le componenti interessate, e che fasi diverse della malattia possono richiedere trattamenti diversi. In alcuni stadi, infatti, è l'infiammazione a svolgere il ruolo più importante nel mantenimento e nella progressione della sintomatologia, in altri è l'iperosmolarità del fluido lacrimale ad essere il principale responsabile della sintomatologia e dello sviluppo del danno istologico, in altri momenti ancora è la carenza di lubrificazione il primo fattore da trattare.

A parte la rimozione dei fattori scatenanti e la terapia specifica delle patologie eventualmente responsabili del quadro, il trattamento della secchezza oculare viene affrontato con uno o più preparati topici, per quanto talvolta si renda necessaria una concomitante terapia sistemica.

I sostituti lacrimali sono i preparati maggiormente utilizzati nel trattamento dell'occhio secco per provvedere alla lubrificazione della superficie oculare e creare un film protettivo; ne esistono diversi tipi dalle caratteristiche anche molto differenti e tutti esercitano un' azione preventiva sulle manifestazioni da irritazione e sulla sintomatologia, pur non possedendo proprietà curative. Da ciò deriva l'importanza cruciale della compliance al trattamento: per un buon risultato terapeutico sono infatti necessarie applicazioni regolari, più o meno frequenti a seconda del tempo di permanenza del preparato sostitutivo, e prolungate nel tempo, poiché nei casi di patologia reversibile sono necessari alcuni mesi prima che la sintomatologia regredisca mentre nelle forme croniche il trattamento non deve più essere sospeso.

I casi più gravi di secchezza oculare sono candidati alla terapia con tappi di collagene (provvisori) o silicone (permanenti) inseriti nei punti lacrimali allo scopo di trattenere il liquido lacrimale, per quanto il rapporto rischi/benefici di questa tecnica sia incerto e debba essere attentamente valutato sul singolo paziente [1].

\section{FARMACOLOGIA}

I carbomeri, anche detti acidi poliacrilici, polimeri carbossivinilici o carbossipolietileni, sono macromolecole ottenute dalla polimerizzazione di acidi acrilici ed eteri, con un peso molecolare compreso tra i 700 e i 4000 $\mathrm{kD}$. Le varie molecole di carbomero, a causa delle forze di repulsione elettrostatica, si dispongono a formare una matrice tridimensionale in grado di trattenere grandi quantità di acqua all'interno, con formazione di un gel stabile, trasparente, incolore e non vischioso. Questo gel, applicato sulla superficie oculare, forma un film lubrificante stabile che non offre resistenza alla chiusura palpebrale e che viene agevolmente trascinato verso 1'alto alla 


\begin{tabular}{|c|c|c|c|c|c|}
\hline \multirow{2}{*}{ Studio } & \multirow{2}{*}{$\begin{array}{l}\text { Tipo di } \\
\text { paziente }\end{array}$} & \multirow{2}{*}{$\begin{array}{l}\text { Disegno } \\
\text { dello studio }\end{array}$} & \multirow{2}{*}{ Confronto } & \multicolumn{2}{|c|}{ Risultati } \\
\hline & & & & $\begin{array}{c}\text { Tempo di } \\
\text { permanenza }\end{array}$ & BUT \\
\hline $\begin{array}{l}\text { Marquardt et } \\
\text { al., } 1986 \text { [8] }\end{array}$ & Volontari sani & $\begin{array}{l}\text { Randomizzato, } \\
\text { OD vs OS }\end{array}$ & Alcol polivinilico & $\begin{array}{l}7 \text { volte (16 vs. } 2 \\
\text { min) superiore } \\
\text { per il carbomero }\end{array}$ & $\begin{array}{l}\text { > con il carbomero; } \\
\text { questo effetto dura } \\
\text { fino a } 6 \text { ore }\end{array}$ \\
\hline $\begin{array}{l}\text { Brewitt, } \\
1994 \text { [9] }\end{array}$ & Volontari sani & $\begin{array}{l}\text { Randomizzato, } \\
\text { OD vs OS }\end{array}$ & $\begin{array}{l}\text { Polivinilpirrolidone } \\
\text { (PVP) }\end{array}$ & $\begin{array}{c}31,7 \pm 10,7 \text { vs } \\
17,4 \pm 6 \text { min } \\
(p<0,0001)\end{array}$ & \\
\hline \multirow{2}{*}{$\begin{array}{l}\text { Al-Mansouri et } \\
\text { al., } 1994 \text { [10] }\end{array}$} & Volontari sani & \multirow{2}{*}{$\begin{array}{l}\text { Randomizzato, } \\
\text { OD vs OS }\end{array}$} & \multirow{2}{*}{$\begin{array}{l}\text { Alcol polivinilico } \\
\text { al } 1,4 \%\end{array}$} & $\begin{array}{l}36,5 \pm 14,2 \text { vs. } \\
16,5 \pm 8,3 \mathrm{~min}\end{array}$ & \multirow{2}{*}{$\begin{array}{l}\text { Dopo } 20 \text { min } \\
\text { significativamente > } \\
\text { per il carbomero }\end{array}$} \\
\hline & Occhio secco & & & $\begin{array}{l}92,8 \pm 35,7 \text { vs. } \\
40,8 \pm 19,4 \mathrm{~min}\end{array}$ & \\
\hline $\begin{array}{l}\text { Wilson et al., } \\
1998 \text { [11] }\end{array}$ & Volontari sani & $\begin{array}{l}\text { OD vs. OS, } \\
\text { farmaci } \\
\text { radiomarcati }\end{array}$ & Soluzione salina & \multicolumn{2}{|c|}{$\begin{array}{l}\text { Dopo } 8 \text { min, ancora presente } 43 \% \text { del } \\
\text { carbomero vs. } 7 \% \text { della salina }\end{array}$} \\
\hline $\begin{array}{l}\text { Troiano et al., } \\
1991 \text { [12] }\end{array}$ & Volontari sani & & Acido ialuronico & \multicolumn{2}{|c|}{$\begin{array}{l}\text { Tempo di ritorno al BUT basale: } 360 \\
\text { min con carbomer vs. } 240 \text { min con } \\
\text { acido ialuronico }\end{array}$} \\
\hline
\end{tabular}

riapertura della palpebra, garantendo un'ottima copertura senza interferire con la meccanica dell' ammiccamento.

Nel corso del processo di gelificazione, la matrice molecolare dei carbomeri assorbe acqua fino a mille volte il proprio volume. Il contatto con gli ioni del liquido lacrimale promuove la liberazione osmotica delle molecole di acqua intrappolate nelle maglie della matrice polimerica, garantendo in tal modo una buona idratazione della superficie oculare. I gel di carbomer, inoltre, possiedono alcune proprietà mucomimetiche dovute all'interazione con le glicoproteine della componente mucinica del liquido lacrimale. Questa interazione, per cui sono stati proposti vari meccanismi (interazione elettrostatica, ponti idrogeno, interazione idrofobica, ecc.) conferiscono ai gel di carbomero caratteristiche bioadesive che ne prolungano il tempo di permanenza sulla superficie oculare.

Alle concentrazioni standard per i prodotti oftalmologici, pari a circa $0,2-0,3 \%$, le proprietà reologiche (elevata viscosità e resistenza al flusso) dei gel di carbomero non consentono il loro imballaggio in flaconi, ma unicamente in tubetti, con perdita dell'accuratezza del dosaggio e della comodità di instillazione. Per due dei gel di carbomero disponibili, tale problema è stato risolto con modificazioni galeniche: la riduzione della concentrazione per il carbopol 940P e l'aggiunta di eccipienti fluidificanti per il carbopol 974P [7].

A causa della struttura macromolecolare, $\mathrm{i}$ carbomeri non attraversano le membrane biologiche e non vengono assorbiti dall'occhio; la loro eliminazione è interamente dovuta al fisiologico ricambio del film lacrimale. Gli studi di farmacocinetica dei gel lacrimali si limitano pertanto a studiarne il tempo di permanenza sulla superficie oculare, parametro considerato strettamente correlato all'azione protettiva, lubrificante e idratante, e pertanto all'efficacia clinica.

La valutazione del tempo di permanenza viene effettuata misurando il tempo di scomparsa della fluorescenza dal film lacrimale dopo somministrazione intraoculare di farmaci marcati con fluorescina. Alcuni autori integrano questa misura obiettiva con un parametro farmacodinamico, mediante valutazione del BUT a intervalli regolari, ma tale metodica soggettiva, che inoltre risente fortemente delle condizioni di illuminazione, non permette di effettuare confronti tra studi differenti.

Le caratteristiche cinetiche dei gel di carbomero sono state confrontate con le lacrime artificiali e l'acido ialuronico, in diverse tipologie di pazienti. I principali risultati di questi lavori sono presentati in Tabella II.

\section{EFFICACIA E TOLLERABILITÀ}

Uno studio in aperto condotto da Danzi e Sfragara [13] ha valutato l'efficacia clinica e la tollerabilità del gel oftalmologico a base di carbomer $974 \mathrm{P}$ al 0,25\% e alcol polivinilico (Siccafluid $®$ - Farmila -Thea Farmaceutici) su 34 pazienti di entrambi i sessi ed età compresa tra i 23 e gli 80 anni (media 55) che presentavano almeno uno dei seguenti sintomi oculari: bruciore, senso di sabbia, fotofobia, secchezza, pesantezza e sensazione di occhio bagnato. Dopo l'arruolamento, i soggetti sono stati trattati per sei mesi con una goccia di gel oftalmico instillata tre volte al giorno. I dati presi in considerazione sono stati la presenza e l'evoluzione temporale della sintomatologia, come riferita dai pazienti attraverso un questionario

\section{Tabella II}

Risultati degli studi di confronto tra gel di carbomero, lacrime artificiali e acido ialuronico 


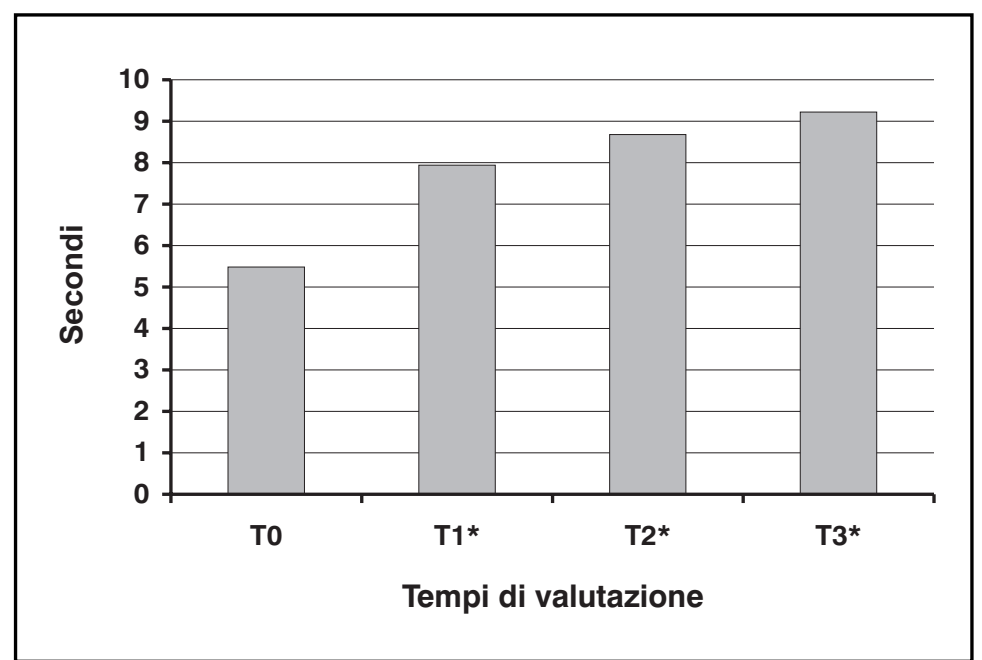

* $=p<0,001$ per il confronto vs. T0

\section{Figura 2}

Valori medi del BUT dell'occhio destro nei vari tempi di valutazione riferiscono la risoluzione o il miglioramento di tutti i sintomi. L'unica eccezione è rappresentata dal mancato miglioramento della sensazione di bruciore in un paziente.

L'andamento dei risultati degli esami obiettivi ha confermato il progressivo miglioramento osservato per la sintomatologia. L'iperemia congiuntivale, presente in 5 pazienti all'arruolamento, al T1era ancora evidenziabile in uno solo, mentre ai controlli successivi era totalmente scomparsa; il numero di pazienti con menisco lacrimale scarso si è progressivamente ridotto, passando da $7(20,6 \%)$ al T0 a 6 $(17,6 \%), 4(12,9 \%)$ e $1(4,3 \%)$ ai T1,T2 e T3, rispettivamente; anche la percentuale di pazienti con lesioni congiuntivali evidenziate dalla fluorescina, pari al 58,8\% (20/34) al basale, si è progressivamente ridotta fino ad annullarsi al T3. Anche l' analisi statistica dei valori del BUT (Figura 2) e del test di Schirmer ha dimostrato un miglioramento progressivo e significativo delle proprietà protettive del film lacrimale.

Cinque pazienti su $34(14,7 \%)$ hanno interrotto il trattamento per la comparsa di bruciore in seguito all'instillazione del collirio. Tale effetto collaterale non si è manifestato immediatamente all'inizio del ciclo terapeutico, bensì dopo alcune settimane, per cui gli autori lo attribuiscono alla sensibilizzazione al benzalconio cloruro, contenuto come conservante nel collirio in studio sebbene in concentrazioni molto basse $(0,006 \%)$.

Per gli altri pazienti, la tollerabilità al trattamento è stata buona nel $90 \%$ dei casi e discreta nei rimanenti [13]

L'efficacia del gel oftalmico a base di carbomero 947Pe PVA (Siccafluid $®)$ nel trattamento dell'occhio secco è stata ulteriormente valutata da Rolando e Contursi in uno studio randomizzato, con disegno a cross-over intrasoggetto e controllato vs. una formulazione convenzionale di sostituto lacrimale a base di ipromellosa [14].

\section{Tabella III}

Prevalenza e andamento temporale della sintomatologia nello studio Danzi e Sfragara (modificato da [13]) dei pazienti, mentre gli altri sintomi indagati presentavano prevalenze minori. L'introduzione della terapia con il gel oftalmico a base di carbomero 947P e PVA ha comportato una importante regressione della sintomatologia già al T1, ulteriormente evidente con la prosecuzione del trattamento, fino ad arrivare al T3, in cui tutti i pazienti rimasti in terapia, meno uno,

\begin{tabular}{|c|c|c|c|c|c|c|c|}
\hline & \multirow[b]{2}{*}{ Sintomo } & \multicolumn{6}{|c|}{ Numero di pazienti (\%) che presenta il sintomo } \\
\hline & & Bruciore & $\begin{array}{c}\text { Senso } \\
\text { di sabbia }\end{array}$ & Secchezza & Fotofobia & Pesantezza & $\begin{array}{c}\text { Occhio } \\
\text { bagnato }\end{array}$ \\
\hline T0 $(n=34)$ & Presente & $26(76,47)$ & $23(67,64)$ & $20(58,82)$ & $12(35,29)$ & $8(23,52)$ & $10(29,41)$ \\
\hline \multirow[t]{3}{*}{$T 1(n=34)$} & Presente, di cui & $16(47,05)$ & $16(47,05)$ & $3(8,82)$ & $4(11,76)$ & $2(5,88)$ & $5(14,70)$ \\
\hline & in forma attenuata & $14(41,18)$ & $13(38,23)$ & $2(5,88)$ & $3(8,82)$ & $1(2,94)$ & $3(8,82)$ \\
\hline & non migliorato & $2(5,88)$ & $3(8,82)$ & $1(2,94)$ & $1(2,94)$ & $0(0,00)$ & $2(5,88)$ \\
\hline \multirow[t]{3}{*}{$T 2(n=31)$} & Presente, di cui & $6(17,35)$ & $7(22,58)$ & $1(3,23)$ & $1(3,23)$ & $1(3,23)$ & $1(3,23)$ \\
\hline & in forma attenuata & $5(16,13)$ & $4(12,90)$ & $1(3,23)$ & $1(3,23)$ & $1(3,23)$ & $1(3,23)$ \\
\hline & non migliorato & $1(3,23)$ & $3(9,68)$ & $0(0,00)$ & $0(0,00)$ & $0(0,00)$ & $0(0,00)$ \\
\hline \multirow[t]{3}{*}{ T3 $(n=23)$} & Presente, di cui & $2(8,70)$ & $1(4,35)$ & $0(0,00)$ & $0(0,00)$ & $0(0,00)$ & $0(0,00)$ \\
\hline & in forma attenuata & $1(4,35)$ & $1(4,35)$ & $0(0,00)$ & $0(0,00)$ & $0(0,00)$ & $0(0,00)$ \\
\hline & non migliorato & $1(4,35)$ & $0(0,00)$ & $0(0,00)$ & $0(0,00)$ & $0(0,00)$ & $0(0,00)$ \\
\hline
\end{tabular}


Per lo studio sono stati reclutati 40 soggetti affetti da sindrome da occhio secco, definita in base alla contemporanea presenza dei seguenti criteri diagnostici:

- Schirmer test $\leq 6 \mathrm{~mm}$ a 5 minuti;

- BUT < 10 secondi;

- Score $\leq 2$ alla colorazione vitale in almeno un settore oculare;

- presenza della sintomatologia caratteristica (bruciore, fastidio all'ammiccamento, sensazione di corpo estraneo, desiderio di tenere gli occhi chiusi).

Dopo l'arruolamento e un periodo di washout di 7 giorni in cui non era ammesso l'utilizzo di alcun lubrificante diverso dalla soluzione fisiologica, i pazienti hanno ricevuto ciascuno dei due farmaci per un ciclo di 10 giorni, separati da un ulteriore periodo di 7 giorni di washout, secondo un ordine stabilito mediante assegnazione randomizzata a due gruppi di trattamento. All'inizio e al termine di ogni ciclo di trattamento sono stati valutati i segni di danno alla superficie oculare, la sintomatologia riferita dai pazienti e il BUT.

Ai pazienti è stato inoltre richiesto di registrare quotidianamente il numero di instillazioni di collirio, per verificare l'assunzione che il prolungato tempo di permanenza sulla superficie oculare del gel di carbomero comporti una minor frequenza di applicazione per il raggiungimento e il mantenimento di un comfort oculare accettabile.

L'analisi della sintomatologia ha messo in evidenza che il singolo paziente che ha iniziato il trattamento con il gel di carbomero ha sempre mostrato un netto miglioramento dei segni e dei sintomi di occhio secco rispetto alle misurazioni basali, miglioramento che non si è ripetuto con il trattamento di controllo; l'inverso è stato osservato nei pazienti assegnati al farmaco di controllo per il primo ciclo terapeutico.

Per ogni sintomo preso in considerazione, gli autori hanno calcolato un miglioramento medio per tipo di trattamento aggregando i dati ottenuti dai pazienti nel corso dei due cicli. Questi risultati, insieme alla frequenza giornaliera media di instillazione dei due farmaci, sono riassunti nella Tabella IV.

Come risulta evidente dalla tabella, il gel oftalmico a base di carbopol 947P+PVA è stato in grado di indurre modificazioni positive $\mathrm{e}$ superiori al farmaco di controllo dello stato della salute oculare e della sintomatologia soggettiva nei pazienti affetti da sindrome da occhio secco.

Questi miglioramenti, inoltre, sono stati ottenuti con un numero inferiore di instillazioni rispetto al sostituto lacrimale convenzionale, con evidenti riflessi positivi sulla qualità di vita.

\begin{tabular}{lcc}
\hline & $\begin{array}{c}\text { Carbomer } \\
\text { 974P + PVA }\end{array}$ & Idromellosa \\
\hline Miglioramento medio & $27 \%$ & $6 \%$ \\
Fastidio oculare & $34 \%$ & $10 \%$ \\
Bruciore & $26 \%$ & $11 \%$ \\
Sensazione di corpo estraneo & $27 \%$ & $12 \%$ \\
Dolore & $31 \%$ & $14 \%$ \\
BUT & $26 \%$ & $5 \%$ \\
Colorazione Rosa Bengala & $27 \%$ & $2 \%$ \\
Colorazione Fluorescina & $28 \%$ & $9 \%$ \\
Complessivo & & \\
& 3,5 & 4,125 \\
\hline
\end{tabular}

Tabella IV

Confronto tra efficacia e consumo di carbopol 947P+PVA e idrossipropilmetilcellulosa nello studio di Rolando e Contursi [14]

\section{COSTIFARMACEUTICI}

Nella Tabella V riportiamo le formulazioni di sostituti lacrimali indicate nel trattamento delle sindromi da occhio secco disponibili in Italia e classificate tra i farmaci. Tali formulazioni differiscono anche notevolmente per principio attivo, tipo di confezionamento e prezzo. Per completezza di trattazione riportiamo inoltre nella Tabella VI i sostituti lacrimali che appartengono alla tipologia merceologica dei parafarmaci.

Il calcolo del costo mensile per ciascun prodotto presentato in Tabella V si è basato sulla valorizzazione di un ipotetico consumo medio di confezioni di prodotto al mese, stimato come segue:

- i contenitori "monodose" sono stati considerati sufficienti per un'applicazione bilaterale, supponendo che ne vengano utilizzati 2 al giorno e che l'eventuale residuo venga gettato;

- i contenitori multiuso da $10 \mathrm{ml}$, corrispondenti a circa 300-400 gocce, sono stati considerati sufficienti a coprire il fabbisogno terapeutico di un paziente con sindrome da occhio secco di grado moderato per un mese di terapia, supponendo che l'eventuale residuo venga gettato, in accordo con le raccomandazioni dei produttori.

È evidente che si tratta di una stima approssimativa che non tiene in conto la notevole variabilità interindividuale della frequenza di instillazione necessaria al raggiungimento del comfort oculare (l'eventuale errore si rifletterebbe in maniera omogenea su tutti i prodotti e non inficerebbe comunque il valore del confronto) e il differente tempo di permanenza sulla superficie oculare dei vari prodotti, risul- 


\begin{tabular}{|c|c|c|c|c|c|c|}
\hline $\begin{array}{l}\text { Principio } \\
\text { attivo }\end{array}$ & Brand & Azienda & Confezione & $\begin{array}{c}\text { Costo confezione } \\
\text { (euro) }\end{array}$ & $\begin{array}{l}\text { Conf/ } \\
\text { mese }\end{array}$ & $\begin{array}{l}\text { Costo } \\
\text { mensile }\end{array}$ \\
\hline \multirow{10}{*}{$\begin{array}{l}\text { Acido } \\
\text { ialuronico }\end{array}$} & Dropstar 0,4\% & Farmigea & 20 cont. $0,5 \mathrm{ml}$ & 11,50 & 3 & 34,50 \\
\hline & Dropyal coll. & Bruschettini & 20 cont. 0,65 ml & 13,69 & 3 & 41,07 \\
\hline & \multirow{3}{*}{ Hyalistil $0,2 \%$} & \multirow{3}{*}{ Sifi } & 20 cont, 0,25 ml & 10,50 & 3 & 31,50 \\
\hline & & & Flac. $10 \mathrm{ml}$ & 12,60 & 1 & 12,60 \\
\hline & & & Flac. $5 \mathrm{ml}$ & 8,50 & 2 & 17,00 \\
\hline & \multirow{2}{*}{ Hy-drop 2mg/ml } & \multirow{4}{*}{$\begin{array}{l}\text { Bausch \& } \\
\text { Lomb Oftal }\end{array}$} & Flac. $10 \mathrm{ml}$ & 11,83 & 1 & 11,83 \\
\hline & & & Flac. 5 ml & 7,44 & 2 & 14,88 \\
\hline & \multirow{2}{*}{ lalurex ipotonico } & & 20 cont. $0,25 \mathrm{ml}$ & 9,66 & 3 & 29,98 \\
\hline & & & 30 con. 0,2 ml & 10,07 & 2 & 20,14 \\
\hline & Irilens collirio & Montefarmaco & Flac. $10 \mathrm{ml}$ & 8,30 & 1 & 8,30 \\
\hline PVA & Hypotears & Medivis & Flac. $10 \mathrm{ml}$ & 9,30 & 1 & 9,30 \\
\hline $\begin{array}{c}\mathrm{BAC}+ \\
\text { carbomer }\end{array}$ & Lacrinorm gel & Farmigea & Tubetto $10 \mathrm{~g}$ & 9,00 & 1 & 9,00 \\
\hline \multirow{3}{*}{$\begin{array}{c}\text { BAC + } \\
\text { ipromellosa }\end{array}$} & Lacrisifi & Sifi & Flac. $10 \mathrm{ml}$ & 8,10 & 1 & 8,10 \\
\hline & Lacrisol & Bruschettini & Flac. $10 \mathrm{ml}$ & 6,10 & 1 & 6,10 \\
\hline & Tirs collirio & Skills in Farmacia & Flac. $10 \mathrm{ml}$ & 5,50 & 1 & 5,50 \\
\hline $\begin{array}{c}\mathrm{BAC}+ \\
\text { metilcellulosa }\end{array}$ & Lacrimart & Baif & Flac. $10 \mathrm{ml}$ & 5,70 & 1 & 5,70 \\
\hline \multirow{5}{*}{ Carbomer } & Siccafluid & Farmila-Thea & Flac. $10 \mathrm{ml}$ & 5,77 & 1 & 5,77 \\
\hline & \multirow{2}{*}{ Dacriogel } & \multirow{2}{*}{$\begin{array}{l}\text { Alcon } \\
\text { Italia }\end{array}$} & Tubetto $10 \mathrm{~g}$ & 10,40 & 1 & 10,40 \\
\hline & & & 30 fiale $0,5 \mathrm{ml}$ & 12,40 & 2 & 28,80 \\
\hline & Dropgel & Eupharmed & Tubetto $10 \mathrm{~g}$ & 8,80 & 1 & 8,80 \\
\hline & Viscotirs & Medivis & Tubetto $10 \mathrm{~g}$ & 9,40 & 1 & 9,40 \\
\hline \multirow{2}{*}{ Carmellosa } & \multirow{2}{*}{ Celluvisc } & \multirow{2}{*}{ Allergan } & $30 \mathrm{fl} .0,5 \%$ & 11,50 & 2 & 23,00 \\
\hline & & & 30 fl. 1,0\% & 12,00 & 2 & 24,00 \\
\hline \multirow{2}{*}{$\begin{array}{l}\text { Destrano + } \\
\text { ipromellosa }\end{array}$} & \multirow{2}{*}{ Dacriosol collirio } & \multirow{3}{*}{$\begin{array}{l}\text { Alcon } \\
\text { Italia }\end{array}$} & Flac. $10 \mathrm{ml}$ & 8,90 & 1 & 8,90 \\
\hline & & & 30 cont. 0,4 ml & 9,90 & 2 & 19,80 \\
\hline Paraffina & Duratirs & & Tubetto $3,5 \mathrm{~g}$ & 10,00 & 1 & 10,00 \\
\hline $\begin{array}{c}\text { Paraffina }+ \\
\text { vaselina }\end{array}$ & Lacrilube & Allergan & Tubetto $3,5 \mathrm{~g}$ & 8,50 & 1 & 8,50 \\
\hline \multirow{3}{*}{ Povidone } & \multirow{2}{*}{ Clarover } & \multirow{2}{*}{$\begin{array}{l}\text { Novartis } \\
\text { Farma }\end{array}$} & Flac. $10 \mathrm{ml}$ & 9,60 & 1 & 9,60 \\
\hline & & & 20 cont. 0,4 ml & 11,90 & 3 & 35,70 \\
\hline & Protagent & Alcon Italia & 20 cont. $0,4 \mathrm{ml}$ & 11,00 & 3 & 33,00 \\
\hline \multirow{2}{*}{$\begin{array}{c}\mathrm{NaCl}+\mathrm{NaHCO} 3+ \\
\mathrm{NaH} 2 \mathrm{PO} 402 \mathrm{H} 2 \mathrm{O}+ \\
\text { MgSO } 4\end{array}$} & \multirow{2}{*}{ Lacrimalfa } & \multirow{2}{*}{$\begin{array}{l}\text { Alfa } \\
\text { Intes }\end{array}$} & Flac. $10 \mathrm{ml}$ & 5,20 & 1 & 5,20 \\
\hline & & & 12 cont. $0,5 \mathrm{ml}$ & 5,20 & 5 & 26,00 \\
\hline Carbomer + tiomersal & Lacrigel & Farmigea & Tubetto $10 \mathrm{~g}$ & 9,00 & 1 & 9,00 \\
\hline
\end{tabular}

$\mathrm{PVA}=$ Alcol polivinilico, $\mathrm{BAC}=$ Benzalconio cloruro

\section{Tabella V}

Confezioni e costi farmaceutici dei sostituti lacrimali indicati nella terapia della sindrome da occhio secco in Italia [Fonte: Informatore Farmaceutico 2004] 


\begin{tabular}{|c|c|c|c|c|}
\hline Principio attivo & Brend & Azienda & Confezione & $\begin{array}{c}\text { Costo confezione } \\
\text { (euro) }\end{array}$ \\
\hline \multirow[t]{2}{*}{ Acido ialuronico-sale sodico } & Blu yal & SOOFT & 15 monodose $0,15 \%$ & 9,80 \\
\hline & & & Coll. $5 \mathrm{ml}$ & 8,30 \\
\hline \multirow[t]{6}{*}{ Seme di tamarindo } & Tsp & FARMIGEA & Sol. oft. $0,5 \% 5 \mathrm{ml}$ & 8,40 \\
\hline & & & Sol. oft. $1 \% 5 \mathrm{ml}$ & 9,80 \\
\hline & & & 20 monodose $0.5 \%$ & 11,00 \\
\hline & & & 20 monodose $1 \%$ & 11,80 \\
\hline & & & Sol. oft. $5 \mathrm{ml} \quad 0,2 \%$ & 7,80 \\
\hline & & & 20 monodose $5 \mathrm{ml} 0,2 \%$ & 10,50 \\
\hline HPGUAR & Systane & ALCON & Coll. $10 \mathrm{ml}$ & 9,90 \\
\hline HPMC & Cellufresh & ALLERGAN & Sol. oft. $12 \mathrm{ml}$ & 13,50 \\
\hline \multirow[t]{2}{*}{ HPMC } & Genteal & NOVARTIS & Coll. $10 \mathrm{ml} 3 \%$ & 9,50 \\
\hline & & & Gel oft. $10 \mathrm{ml} \mathrm{3 \%}$ & 10,40 \\
\hline Acido ialuronico-sale sodico & Hylo comod & VISUFARMA & Flac. $10 \mathrm{ml}$ senza conservanti & 13,00 \\
\hline Acido ialuronico+Elettroliti & Oxyal & TUBILUX & Coll. 10ml & 12,90 \\
\hline Soluzione salina & Hydrabak & FARMILA THEA & Flac. ABAK $10 \mathrm{ml}$ senza conservanti & 8,50 \\
\hline \multirow[t]{2}{*}{ Acido ialuronico-sale sodico } & Blu gel & SOOFT & Coll. $5 \mathrm{ml}$ & 8,90 \\
\hline & & & 15 monodose $0.35 \mathrm{ml}$ & 10,20 \\
\hline HPMC $1 \%$ & Cellumed & ALLERGAN & Coll. $15 \mathrm{ml}$ & 13,50 \\
\hline PVP 2\%+ elettroliti & Filmabak & FARMILA THEA & Flac. ABAK $10 \mathrm{ml}$ senza conservanti & 9,50 \\
\hline \multirow[t]{2}{*}{ Acido ialuronico-sale sodico } & Hyabak & FARMILA THEA & Flac. ABAK $10 \mathrm{ml}$ senza conservanti & 11,50 \\
\hline & Next 300 & BIOOS I. & Coll. $5 \mathrm{ml}$ & 7,50 \\
\hline \multirow[t]{2}{*}{ Acido ialuronico-sale sodico } & Hyluprotect & FARMILA THEA & Coll. $10 \mathrm{ml}$ & 6,20 \\
\hline & & & 15 monodosi $25 \%$ & 8,30 \\
\hline HPMC & Next & BIOOS I. & 20 monodose $0.35 \mathrm{ml}$ & 11,00 \\
\hline soluzione salina & Blu sal & SOOFT & 15 monodose $0.35 \mathrm{ml}$ & 8,60 \\
\hline HPMC & Vistil & TUBILUX & Coll. $10 \mathrm{ml}$ & 10,60 \\
\hline Acido ialuronico-sale sodico & Vismed & TUBILUX & 20 monodose $0,3 \mathrm{ml}$ & 9,00 \\
\hline Carbossimetilcellulosa 0,25\%+El. & Theratears & EUPHARMED & 24 monodose $0,6 \mathrm{ml}$ & 13,50 \\
\hline HPMC & Lacrilens & TUBILUX & Coll. $10 \mathrm{ml}$ & 12,00 \\
\hline Acido ialuronico-sale sodico & Fermavisc & MEDIVIS & 20 monodose $0,3 \mathrm{ml}$ & 13,00 \\
\hline HPMC & Lacrilux I & TUBILUX & Coll. $10 \mathrm{ml}$ & 7,80 \\
\hline PVP2\% & Wet comod & VISUFARMA & Coll. $10 \mathrm{ml}$ & 9,50 \\
\hline
\end{tabular}

\section{Tabella VI}

Confezioni e costi dei sostituti lacrimali classificati tra i parafarmaci [Fonte: Informatore Farmaceutico 2004]

tando pertanto conservativa a sfavore delle molecole più efficaci, come i gel di carbomer.

Come si evince dal prospetto, il gel a base di carbopol 974P e PVA (Siccafluid $囚)$ è il prodotto caratterizzato dal minor costo/confezione e dal minor costo mensile tra le formulazioni a base di carbomer disponibili in Italia (con un risparmio medio mensile oscillante tra i 3 e i 6 euro, a seconda del prodotto usato per il confronto) ed è uno dei meno cari anche nel complesso dei sostituti lacrimali disponibili in Italia.

Per quanto la carenza di studi clinici di confronto tra le varie molecole e formulazioni di sostituti lacrimali non consenta una va- 


\section{Tabella VIla}

Analisi dei valori nel paziente Sjögren: confronto vs. placebo

\section{Tabella VIIb}

Analisi dei valori nel paziente Sjögren: confronto vs. altri sostituti lacrimali

\section{Tabella VIIc}

Analisi dei valori nel paziente non Sjögren: confronto vs. placebo

\section{Tabella VIId}

Analisi dei valori nel paziente non Sjögren: confronto vs. altri sostituti lacrimali

\begin{tabular}{|c|c|c|}
\hline Valore & Clinico & Economico \\
\hline Positivo & $\begin{array}{l}\text { Efficace controllo della sintomatologia } \\
\text { nella maggioranza dei casi } \\
\text { Riduzione delle complicazioni } \\
\text { Miglioramento della qualità di vita }\end{array}$ & $\begin{array}{l}\text { Riduzione della perdita di produttività per } \\
\text { disabilità } \\
\text { Riduzione dei costi per la cura delle } \\
\text { complicazioni }\end{array}$ \\
\hline Negativo & Casi di intolleranza & - \\
\hline
\end{tabular}

\begin{tabular}{cll}
\hline Valore & \multicolumn{1}{c}{ Clinico } & Economico \\
\hline Positivo & $\begin{array}{l}\text { Efficacia paragonabile o superiore } \\
\text { Miglioramento della qualità di vita per } \\
\text { ridotta frequenza di instillazione } \\
\text { Negativo }\end{array}$ & Nessun costo farmaceutico \\
& Casi di intolleranza & - \\
\hline
\end{tabular}

\begin{tabular}{|c|c|c|}
\hline Valore & Clinico & Economico \\
\hline Positivo & $\begin{array}{l}\text { Efficace controllo della sintomatologia } \\
\text { nella maggioranza dei casi } \\
\text { Riduzione delle complicazioni } \\
\text { Miglioramento della qualità di vita }\end{array}$ & $\begin{array}{l}\text { Riduzione della perdita di produttività per } \\
\text { disabilità } \\
\text { Riduzione dei costi per la cura delle } \\
\text { complicazioni }\end{array}$ \\
\hline Negativo & Casi di intolleranza & Costo del farmaco \\
\hline
\end{tabular}

\begin{tabular}{|c|c|c|}
\hline Valore & Clinico & Economico \\
\hline Positivo & $\begin{array}{l}\text { Efficacia paragonabile o superiore } \\
\text { Miglioramento della qualità di vita per } \\
\text { ridotta frequenza di instillazione }\end{array}$ & $\begin{array}{l}\text { Costo farmaceutico uguale o minore dei } \\
\text { concorrenti }\end{array}$ \\
\hline Negativo & Casi di intolleranza & - \\
\hline
\end{tabular}

lutazione metodologicamente corretta della convenienza farmacoeconomica ad utilizzare un particolare sostituto lacrimale nella terapia dell'occhio secco, l'ottimo profilo di efficacia e tollerabilità e il costo relativamente basso del gel a base di carbopol 974P e PVA rispetto ai competitors suggeriscono che questo prodotto si caratterizzi per uno dei migliori rapporti costo/efficacia in quest' ambito di utilizzo.

Le medesime considerazioni sono molto probabilmente alla base della decisione del SSN di collocare il prodotto, unico esempio in questa classe farmaceutica, in fascia A, a totale carico del SSN, per quanto riguarda la terapia dell'occhio secco nei pazienti affetti da sindrome di Sjögren primaria o secondaria (nota AIFA 83).

\section{QUALITÀ DI VITA E COMPLIANCE}

Per quanto si tratti di una patologia generalmente benigna, la sindrome da occhio secco, se non adeguatamente trattata, comporta un elevato costo per il paziente in termini di qualità di vita, interferendo con le comuni attività e causando un costante fastidio o dolore.
Ogni trattamento efficace sulla sintomatologia oculare comporta pertanto un miglioramento della qualità di vita del paziente. Il valore che il paziente con sindrome da occhio secco attribuisce al proprio benessere oculare non è stato indagato da studi specifici condotti al fine di valutare il parametro farmacoeconomico più idoneo alla definizione del valore soggettivo di un trattamento puramente sintomatologico, ossia la disponibilità a pagare (WTP - willingness to pay) per ottenere un determinato miglioramento. Tuttavia, appare assai ragionevole che una spesa di circa 6 euro/mese per la riduzione o risoluzione sintomatologica sia accettabile dalla maggioranza dei pazienti, che d'altronde non dispongono di alternative meno costose.

Un ulteriore miglioramento della qualità di vita indotto dall'utilizzo del gel a base di carbopol 974P e PVA, rispetto ai farmaci concorrenti, è dipendente dalle caratteristiche di praticità d'impiego del prodotto, confezionato in flaconi di collirio anziché nel classico tubetto, e dalla ridotta frequenza di instillazione giornaliera. La riduzione del numero di applicazioni quotidiane, oltre a influenzare positivamente la qualità di vita del paziente, è inoltre un 
ben noto fattore favorente la compliance, che è parametro di fondamentale importanza per garantire l'efficacia di un trattamento, soprattutto nel caso di terapie croniche e preventive come quella della sindrome da occhio secco.

\section{CONCLUSIONI: IL VALORE DEL GEL A BASE DI CARBOMERO 974P E PVA}

Abbiamo già accennato al fatto che il tipo di dati scientifici ed economici disponibili sull'utilizzo dei gel di carbomero nella terapia dell'occhio secco sono insufficienti per una valutazione rigorosa delle loro prestazioni farmacoeoconomiche, che richiederebbe il disegno e la conduzione di studi di confronto che contemplino anche dei parametri economici e di soddisfazione del paziente.

Ciò tuttavia non significa che si debba rinunciare all'inquadramento farmacoeconomico di questi prodotti. Una tecnica di valutazione farmacoeconomica preliminare, utile a individuare le diverse ricadute potenziali di un trattamento, è la cosiddetta "analisi dei valori".

Poiché le ricadute possono essere diverse per i vari attori coinvolti nelle decisioni sanitarie, sia a livello di utilità che a livello di costi, tale tecnica prevede la costruzione della matrice dei valori, una serie di tabelle $2 \times 2$ che esplicita conseguenze positive e negative di un'alternativa nei due livelli di valutazione, clinico ed economico, per ogni punto di vista rilevante.

Nella Tabella VII proponiamo una matrice dei valori, costruita in base alle considerazioni cliniche ed economiche già esposte, in cui vengono inquadrate le conseguenze del trattamento con il gel di carbomero 974P e PVA nei pazienti con occhio secco associato o meno a sindrome di Sjögren e rispetto a placebo e ad altri sostituti lacrimali.

\section{BIBLIOGRAFIA}

1 American Academy of Ophthalmology Dry Eye Syndrome-Preferred Practice Pattern, 2003, American Academy of Ophthalmology http://www.aao.org/education/library/ppp/dryeye_new.cfm

2 Hikichi T, Yoshida A, Fukui Y, et al. Prevalence of dry eye in Japanese eye centers. Graefes Arch Clin Exp Ophthalmol 1995;223:555-8

3 Schein OD, Munoz B, Tielsch JM, et al. Prevalence of dry eye among the elderly. Am J Ophthalmol 1997;124:723-8.

4 McCarty CA, Bansal AK, Livingston PM, et al. The epidemiology of dry eye in Melbourne, Australia. Ophthalmology 1998;105:1114-9

5 Moss SE, Klein R, Klein BE. Prevalence of and risk factors for dry eye syndrome. Arch Ophthalmol 2000;118:12648.

6 Rolando M e Calabria G. Il film lacrimale Fogliazza editore, Milano 1997.

7 Pouliquen P. Carbomer gels in dry eye treatment: literature review. J Fr Ophthalmol 1999;22(8):903-13

8 Marquardt R Die Behandlung des trockenen Auge mit einem neuen tropffaehigen Gel. Klin Mbl Augenheilkd 1986;189:51-4

9 Brewitt H Traenenersatzmittel: experimentelle und klinische Beobachtungen. Klin Mbl Augenheilkd 1988;193:27582

10 Al-Mansouri S, Tabbara KF, Rask-Pedersen E Lubrithal (Leo viscous eye gel), precorneal residence time in normal and dry eyes. Doc. Ophthalmol 1994;88:187-94 
11 Wilson CG, Zhu YP, Frier M, et al. Ocular contact time of a carbomer gel (GelTears) in humans. Br J Ophthamol

12 Troiano P, Calabrese A, Aschero M, Miglior M The possibility to stabilize the tear film: comparison between two tear substitutes. In: Van Bijsterveld OP, Lemp MA, SpinelliD The lacrimal system - Symposium, 1990 March 17; Singapore, Malaysia. Amsterdam: Kugler \& Ghedini, 1991 pp 97-101.

13 Danzi P e Sfagara I Efficacia e tollerabilità di un collirio a base di carbopol 974P 0,25\% e PVA(Siccafluid) L'oftalmologo informer Hippocrates 14:50-6

14 Rolando M e Contursi D Efficacia del preparato Carbopol 974p e Alcool Polivinilico(Siccafluid) nel trattamento della sindrome da occhio secchio L'oftalmologo informer Hippocrates 15:32-6

15 L'Informatore Farmaceutico 2004, OEMF, Milano 